\title{
A GROUP-BLIND MULTIUSER RECEIVER FOR MC-CDMA SYSTEMS
}

\author{
H. Cheng and S. C. Chan
}

\author{
Dept. of Electrical \& Electronic Engineering \\ The University of Hong Kong, Pokfulam Road, Hong Kong
}

\begin{abstract}
In this paper, a group-blind multiuser receiver is investigated for multicarrier code division multiple access (MC-CDMA) systems. In uplink transmissions, the receiver has the knowledge of the spreading sequences of the users within the cell, while the spreading sequences of the users from other cells are unknown. By imposing linear vector constraints on the multiuser detector, group-blind detection can efficiently suppress the known interferers, while mitigating the unknown interferers based on the minimum mean square error (MMSE) principle. A subspacebased channel estimation algorithm is proposed to blindly estimate the channel information. An adaptive implementation of the group-blind multiuser detection is also developed by using signal and noise subspace tracking algorithms. Numerical results show that the performance of the group-blind detector is much better than the subspace-based blind linear MMSE detector.
\end{abstract}

\section{INTRODUCTION}

MC-CDMA was recently proposed as an efficient multicarrier transmission scheme for supporting multiple access communications [1]. It combines CDMA and orthogonal frequency division multiplexing (OFDM) techniques. It has received considerable attention because of its advantages in frequency diversity, multipath fading resilience and etc [2].

Similar to conventional CDMA systems, MC-CDMA is an interference-limited system. It is rather sensitive to multiple access interference (MAI), which arises from the other users signals transmitted using the same frequency band. Recently, a subspace-based blind MMSE detection has been proposed in [3] for MAI suppression in CDMA systems. By estimating signal subspace from the received signals, the linear MMSE detector can be blindly obtained with the known spreading sequence of the desired user. This method is especially attractive for the CDMA downlink transmissions, where the receiver only knows its own spreading sequence. However, in uplinks, the base station receiver has the knowledge of the users within the cell, but not that of the users from other cells. In [4], subspace-based group-blind multiuser detection is proposed for CDMA uplinks, which zero-forces the interferers within the cell, while suppresses the unknown interferers from other cells with the MMSE principle. By imposing linear vector constraints, the group-blind multiuser detection considerably outperforms the subspace-based blind method in CDMA uplinks.

In [5] [6], subspace-based blind detection and channel estimation techniques have been proposed for MC-CDMA downlinks. In this paper, the group-blind receiver in MC-CDMA uplinks is studied. To investigate the performance of the groupblind and blind detection in practical applications, the adaptive implementation of the two detectors is developed based on the signal and noise subspace trackers. A large number of subspace trackers with various complexities and performance have been proposed [7]. By computational complexity, subspace tracking algorithms can be classified in $O\left(M^{2} r\right), O\left(M r^{2}\right)$ and $O(M r)$ techniques, where $M$ is the input vector dimension and $r$ is the number of eigenvectors to be tracked. In particular, the biiteration square-root SVD subspace tracking algorithm (Bi-SVD) [8] with a complexity of $O\left(M r_{s}^{2}\right)$ ( $r_{s}$ is the rank of signal subspace), and the projection approximation subspace tracking with deflation (PASTd) algorithm [9] with a complexity of $O\left(M r_{s}\right)$ are used for tracking principle eigenvectors and eigenvalues; while the square-root $\mathrm{QR}$ inverse iteration method [10] with a complexity $O\left(M r_{n}^{2}\right)$ ( $r_{n}$ is the rank of noise subspace, and $r_{s}+r_{n}=M$ ) is adopted for minor subspace tracking. In the numerical simulations, we found that the convergence of the PASTd algorithm is quite slow when the rank of signal subspace is large and input signal-to-noise ratio (SNR) is high. Whereas, the Bi-SVD signal subspace tracker presents performance close to the solution with exact SVD.

The rest of the paper is organized as follows: Section 2 describes the principle of MC-CDMA systems. Section 3 is devoted to the group-blind multiuser receiver, while the Bi-SVD signal subspace tracking algorithm is presented in Section 4. Section 5 gives the numerical examples, and finally, conclusion is drawn in Section 6.

\section{SYSTEM MODEL}

We consider a MC-CDMA system for uplink transmission from the terminal users to the base station. Fig. 1 shows the structure of an MC-CDMA transmitter. The original data stream of the $k t h$ user is first converted into $P$ parallel data sequences. Each serial/ parallel $(\mathrm{S} / \mathrm{P})$ converted output spreads with a spreading sequence of length $M$. The data chips after spreading are $\mathrm{S} / \mathrm{P}$ converted into $M$ parallel subcarriers. The total $N=P M$ chips are modulated by the IDFT and converted back into serial data. The cyclic prefix is inserted between symbols to avoid intersymbol interference caused by multipath fading. Let $T$ be the symbol duration of the original data stream, and $T_{S}$ be the one at the subcarrier, then it has $T_{s}=P T$. The frequency separation between the successive subcarriers is $\Delta f=1 / T_{S}$. The complex 
equivalent transmitted signal of user $k$ can be written as

$$
s_{k}(t)=\sum_{i=-\infty}^{i} \sqrt{P_{k}} \sum_{p=0}^{P-1} \sum_{m=0}^{M-1} b_{k, p}^{(i)} c_{k, m}^{(i)} \psi\left(t-i T_{s}\right) e^{j 2 \pi(P m+p) \Delta f t},
$$

where $P_{k}$ is the chip energy, $b_{k, p}^{(i)}$ is the transmitted $i$ th data bit in the $p$ th data stream, $c_{k, m}^{(i)}$ is the $m$ th chip of the given spreading sequence, and $\psi(t)$ represents the rectangular pulse waveform. Note that there are $P$ data streams for the $k t h$ user, and the data bits on different streams use the same spreading sequence. In order to achieve the maximum frequency diversity, the subcarrier separation for $b_{k, p}^{(n)}$ is $\Delta f^{\prime}=P / T_{s}$. The kth user's signal $s_{k}(t)$ propagates through a frequency-selective Rayleigh fading channel with $L$ received paths. The channel is represented by a time-variant impulse response

$$
g_{k}(t)=\sum_{l=1}^{L} \alpha_{k, l}(t) \delta\left(t-\tau_{k, l}\right),
$$

where $k$ is the user index, $\alpha_{k, l}(t)$ is the lth path gain which is independent zero mean, complex Gaussian random process, and $\tau_{k, l}$ is the propagation delay for the lth path, respectively.

In MC-CDMA systems, because of the large symbol duration, each subcarrier approximately experiences flat fading. Assume that there are $K$ simultaneous users in the system, all using the same selection of $P$ and $M$. At the receiver, the signal is sampled at a rate $N / T_{S}$, the samples corresponding to the cyclic prefix are discarded, and a DFT of size $N$ is performed. For simplicity, we will consider a synchronous model, where all the users arrive at the same time. The received signal $\mathbf{y}_{p}(i)=\left[y_{p}(i), \cdots, y_{P \cdot(M-1)+p}(i)\right]^{T}$ after DFT operation with respect to the data symbol $b_{k, p}^{(i)}$ can be given as

$$
\mathbf{y}_{p}(i)=\sqrt{P_{k}} \sum_{k=0}^{K-1} \mathbf{C}_{k} \mathbf{h}_{k, p}^{(i)} b_{k, p}^{(i)}+\mathbf{n}(i)=\sum_{k=0}^{K-1} \sqrt{P_{k}} \mathbf{d}_{k, p}^{(i)} b_{k, p_{k}}^{(i)}+\mathbf{n}(i),
$$

where (for convenience, the subscript $i$ is omitted) $b_{k, p}$ is the data symbol corresponding to the pth data stream; $P_{k}$ is the chip energy; $\mathbf{h}_{k, p}$ denotes channel frequency response, whose element is $h_{k, P m+p}=\frac{1}{\sqrt{N}}\left(\sum_{l=0}^{L-1} \alpha_{k, l} e^{-\frac{j 2 \pi l(P m+p)}{N}}\right), m=0, \ldots, M-1$; $\mathbf{C}_{k}=\operatorname{diag}\left\{c_{k}[0], c_{k}[1], \ldots, c_{k}[M-1]\right\}$ is the code matrix; $\mathbf{d}_{k, p}$ $=\left[c_{k}^{0} h_{k, p}, \cdots, c_{k}^{M-1} h_{k, P(M-1)+p}\right]^{T}$ denotes the effective signature; and $\mathbf{n}=\left[\eta_{p}, \eta_{P+p}, \cdots, \eta_{P(M-1)+p}\right]^{T}$ represents complex additive white Gaussian with zero mean and variance $\sigma_{v}^{2}$.

\section{GROUP-BLIND MULTIUSER DETECTION}

Assume that the data stream for each user is independent, i.e., $E\left\{b_{i}(t) b_{j}^{*}(t)\right\}=0$, and the ambient noise is white. Hence, referring to the linear model in Eq. (3), the data correlation

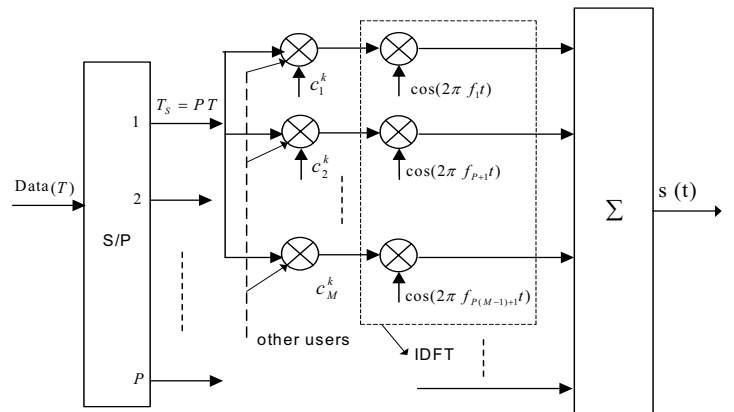

Fig. 1. Transmitter structure for MC-CDMA.

matrix $\mathbf{R}_{\mathbf{y}}$ of the received signal can be given by

$$
\mathbf{R}_{\mathbf{y}}=E\left\{\mathbf{y}(n) \mathbf{y}^{H}(n)\right\}=\sum_{k=0}^{K-1} P_{k} \mathbf{d}_{\mathbf{k}} \mathbf{d}_{\mathbf{k}}^{\mathbf{H}}+\sigma_{n}^{2} \mathbf{I}_{M \times M},
$$

Following the eigenvalue decomposition, $\mathbf{R}_{\mathbf{y}}$ is given as

$$
\begin{aligned}
\mathbf{R}_{\mathbf{y}} & =\mathbf{U} \boldsymbol{\Lambda} \mathbf{U}^{H}=\left[\begin{array}{ll}
\mathbf{U}_{s} & \mathbf{U}_{n}
\end{array}\right]\left[\begin{array}{cc}
\boldsymbol{\Lambda}_{s} & \mathbf{0} \\
\mathbf{0} & \boldsymbol{\Lambda}_{n}
\end{array}\right]\left[\begin{array}{c}
\mathbf{U}_{s}^{H} \\
\mathbf{U}_{n}^{H}
\end{array}\right] \\
& =\mathbf{U}_{s} \boldsymbol{\Lambda}_{s} \mathbf{U}_{s}^{H}+\mathbf{U}_{n} \boldsymbol{\Lambda}_{n} \mathbf{U}_{n}^{H},
\end{aligned}
$$

where $\boldsymbol{\Lambda}_{\mathbf{s}}=\operatorname{diag}\left(\lambda_{1}, \lambda_{2}, \ldots \lambda_{K}\right)$ contains $K$ largest eigenvalues of $\mathbf{R}_{\mathbf{y}}$, which corresponds to the $K$ active users, $\mathbf{U}_{\mathbf{s}} \in \mathbf{C}^{M \times K}$ contains $K$ signal eigenvectors which span the signal subspace, and $\mathbf{U}_{n} \in \mathbf{C}^{M \times(M-K)}$ contains $M-K$ noise eigenvectors which span the noise subspace.

Consider an uplink transmission, where the base station receiver has the knowledge of the users within the cell, while the spreading sequences of users from other cells are unknown. In group-blind multiuser detection, it is assumed that the given spreading sequences of the first $\hat{K}$ users, $\hat{K} \leq K$, are known to the receiver. Denote $\mathbf{D}=\left[\mathbf{d}_{0}, \cdots, \mathbf{d}_{\hat{K}-1}\right]$ and $\mathbf{f}_{0}=[1,0 \cdots, 0]^{T}$, where $\mathbf{f}_{0} \in \mathfrak{R}^{\hat{K} \times 1}$. Without loss of generality, the subscript $p$ will be dropped from the model in Eq. (3), and user 1 is the desired user. Then the group-blind linear MMSE detector for user 1 can be formulated as the following multiple constrained optimization problem:

$$
J(\mathbf{w})=\min _{w} E\left\{\left|\sqrt{P_{0}} b_{0}(n)-\mathbf{w}^{H} \mathbf{y}(n)\right|^{2}\right\} \text {, s.t. } \mathbf{w}^{H} \mathbf{D}=\mathbf{f}_{0}^{T} .
$$

By the method of Lagrange multipliers, the solution of Eq. (6) can be written as [4]

$$
\mathbf{w}=\mathbf{U}_{s} \boldsymbol{\Lambda}_{s}^{-1} \mathbf{U}_{s}^{H} \mathbf{D} \cdot\left(\mathbf{D}^{H} \mathbf{U}_{s} \boldsymbol{\Lambda}_{s}^{-1} \mathbf{U}_{s}^{H} \mathbf{D}\right)^{-1} \mathbf{f}_{0} .
$$

If only the spreading sequence of desired user is known to the receiver, it has $\mathbf{D}=\mathbf{d}_{0}$ and $\mathbf{f}_{0}=1$. Consequently, the subspacebased blind MMSE detector is formulated as

$$
J(\mathbf{w})=\min _{w} E\left\{\left|\sqrt{P_{0}} b_{0}(n)-\mathbf{w}^{H} \mathbf{y}(n)\right|^{2}\right\} \text {, s.t. } \mathbf{w}^{H} \mathbf{d}_{0}=1 .
$$

Then the linear detector $\mathbf{w}$ can be solved as [3]

$$
\mathbf{w}=\frac{1}{\mathbf{d}_{0}^{H} \mathbf{U}_{s} \boldsymbol{\Lambda}_{s}^{-1} \mathbf{U}_{s}^{H} \mathbf{d}_{0}} \mathbf{U}_{s} \boldsymbol{\Lambda}_{s}^{-1} \mathbf{U}_{s}^{H} \mathbf{d}_{0}
$$


A blind subspace-based channel estimation algorithm is developed as follows. Consider a channel with $L$ multipaths, and $\mathbf{g}_{k}$ is the channel impulse response of the kth user. It has $\mathbf{h}_{k}=\mathbf{F}_{m} \mathbf{g}_{k}$, where $\mathbf{F}_{m} \in \mathbf{C}^{M \times L}$ is a DFT matrix. We then have $\mathbf{d}_{k}=\mathbf{C}_{k} \mathbf{F}_{m} \hat{\mathbf{g}}_{k}$, where $\mathbf{C}_{k}$ is the code matrix, and $\hat{\mathbf{g}}_{k}$ denotes the estimation of $\mathbf{g}_{k}$. The orthogonality between the noise and signal subspace implies $\mathbf{U}_{n}^{H} \mathbf{d}_{k}=\mathbf{U}_{n}^{H} \mathbf{C}_{k} \mathbf{F}_{m} \mathbf{g}_{k}=0$. Then the channel vector $\mathbf{g}_{k}$ can be estimated by least-squares approach:

$$
\hat{\mathbf{g}}_{k}=\arg \min _{\mathbf{g}_{k} \in \mathbf{C}^{L \times 1}} \mathbf{g}_{k}^{H} \underbrace{\left\{\mathbf{F}_{m}^{H} \mathbf{C}_{k}^{H} \mathbf{U}_{n} \mathbf{U}_{n}^{H} \mathbf{C}_{k} \mathbf{F}_{m}\right.}_{\mathbf{\Omega}_{k}}\} \mathbf{g}_{k} .
$$

It is seen that $\mathbf{g}_{k}$ is the eigenvector corresponding to the smallest eigenvalue of matrix $\mathbf{\Omega}_{k}$. If $\left[\mathbf{C}_{0} \mathbf{F}_{m}, \mathbf{d}_{1}, \mathbf{d}_{2}, \cdots, \mathbf{d}_{\hat{K}-1}\right]$ has full column rank, the channel estimation is unique and exact up to a complex constant. The residual scalar ambiguity inherent in blind algorithms can be resolved by transmitting short pilot symbols or using differential encoding/decoding.

\section{BI-SVD SIGNAL SUBSPACE TRACKING}

We adopted Bi-SVD and PASTd subspace tracking algorithms to recursively estimate signal subspace. It is found that the convergence of the PASTd is rather slow. While the Bi-SVD subspace tracker presents much faster convergence, and its performance is very close to the solution using exact SVD. In this section, Bi-SVD algorithm is presented for signal subspace tracking. Let a $(n+1) \times M$ data matrix $\mathbf{Y}^{H}(n)=[\mathbf{y}(0) \cdots \mathbf{y}(n)]$ denote received signals at the first $n$ data samples. Define a $(n+1) \times(n+1)$ matrix $\Lambda(n)=\operatorname{diag}\left\{\lambda^{n}, \lambda^{n-1}, \cdots 1\right\}$, then the exponentially windowed data sample covariance matrix $\mathbf{R}_{\mathbf{y}}$ is

$$
\begin{aligned}
\mathbf{R}_{\mathbf{y}}(n) & =\sum_{i=0}^{n} \lambda^{n-i} \mathbf{y}(i) \mathbf{y}^{H}(i) \\
& =[\mathbf{\Lambda}(n) \mathbf{Y}(n)]^{H}[\mathbf{\Lambda}(n) \mathbf{Y}(n)]=\mathbf{X}^{H}(n) \mathbf{X}(n),
\end{aligned}
$$

where $\mathbf{X}(n)=\left[\begin{array}{c}\mathbf{y}^{H}(n) \\ \sqrt{\lambda} \mathbf{X}(n-1)\end{array}\right]$. Let $\mathbf{Q}_{A}(0)=\left[\begin{array}{l}\mathbf{I}_{r_{s}} \\ \mathbf{0}\end{array}\right]$. Consider the following bi-iteration applied on the data matrix $\mathbf{X}(n)$ : [8]

$$
\begin{aligned}
& \mathbf{B}(n)=\mathbf{X}(n) \mathbf{Q}_{A}(n-1), \\
& \mathbf{B}(n)=\mathbf{Q}_{B}(n) \mathbf{R}_{B}(n):(n+1) \times r_{s} Q R-\text { factorization, } \\
& \mathbf{A}(n)=\mathbf{X}^{H}(n) \mathbf{Q}_{B}(n-1), \\
& \mathbf{A}(n)=\mathbf{Q}_{A}(n) \mathbf{R}_{A}(n): M \times r_{s} Q R-\text { factorization. }
\end{aligned}
$$

It can be shown that $\mathbf{Q}_{A}(n)$ will converge toward the $r_{S}$ dominant right eigenvectors of $\mathbf{X}(n)$, which is identical to the signal subspace basis matrix of the covariance matrix $\mathbf{R}_{\mathbf{y}}$. However, the vertical dimension of the data matrix $\mathbf{X}(n)$ keeps increasing during the updating process. In order to eliminate the growing matrices in Eq. (12), a key step in the bi-iteration subspace tracker is to approximate the data matrix $\mathbf{X}(n)$ with a low-rank approximation $\widetilde{\mathbf{X}}(n)$, i.e.,

$$
\tilde{\mathbf{X}}(n)=\mathbf{Q}_{B}(n) \mathbf{R}_{B}(n) \mathbf{Q}_{A}{ }^{H}(n-1) .
$$

This approximation leaves the auxiliary recursion matrix $\mathbf{B}(n)$ in Eq. (12) unaltered: $\mathbf{B}(n)=\mathbf{X}(n) \mathbf{Q}_{A}(n-1)=\widetilde{\mathbf{X}}(n) \mathbf{Q}_{A}(n-1)$. A square-root bi-iteration subspace tracking algorithm is summarized in Table 1. More details about the bi-iteration subspace tracking algorithm can be referred to [8].

TABLE 1

A SQUARE-ROOT BI-ITERATION SUBSPACE TRACKER

$$
\begin{aligned}
& \text { Initialize }: \mathbf{Q}_{A}(n-1)=\left[\begin{array}{c}
\mathbf{I}_{s} \\
\mathbf{0}
\end{array}\right] ; \mathbf{R}_{B}(n-1)=\boldsymbol{\theta}_{A}(n-1)=\mathbf{I}_{r_{s}} ; \\
& \text { Update }: \mathbf{h}(n)=\mathbf{Q}_{A}^{H}(n-1) \mathbf{y}(n), \overline{\mathbf{y}}(n)=\mathbf{y}(n)-\mathbf{Q}_{A}(n-1) \mathbf{h}(n), \\
& e_{y}(n)=\|\overline{\mathbf{y}}(n)\|_{2}, \overline{\mathbf{y}}(n)=\overline{\mathbf{y}}(n) / e_{y}(n), \\
& \mathbf{H}(n)=\mathbf{R}_{B}(n-1) \mathbf{Q}_{A}(n-1),\left[\begin{array}{c}
\mathbf{R}_{B}(n) \\
\mathbf{0}
\end{array}\right]=\mathbf{G}_{B}(n)\left[\begin{array}{l}
\mathbf{h}^{H}(n) \\
\mathbf{H}(n)
\end{array}\right], \\
& \mathbf{h}_{R}^{H}(n) \mathbf{R}_{B}(n)=\mathbf{h}^{H}(n): \text { back substitution to solve } \mathbf{h}_{R}^{H}(n), \\
& \mathbf{H}_{R}^{H}(n) \mathbf{R}_{B}(n)=\mathbf{H}(n): \text { back substitution to solve } \mathbf{H}_{R}(n), \\
& {\left[\begin{array}{c}
\mathbf{R}_{A}(n) \\
\mathbf{0}
\end{array}\right]=\mathbf{G}_{A}(n)\left[\begin{array}{l}
\lambda \mathbf{R}_{A}(n-1) \mathbf{H}_{R}(n)+\mathbf{h}(n) \mathbf{h}_{R}^{H}(n) \\
e_{y}(n) \mathbf{h}_{R}^{H}(n)
\end{array}\right],} \\
& \mathbf{G}_{A}(n)=\left[\begin{array}{cc}
\boldsymbol{\theta}_{A}(n) & * \\
\mathbf{f}^{H}(n) & *
\end{array}\right]: \quad \text { extract } \boldsymbol{\theta}_{A}(n) \text { and } \mathbf{f}^{H}(n), \\
& \mathbf{Q}_{A}(n)=\mathbf{Q}_{A}(n-1) \boldsymbol{\theta}_{A}(n)+\overline{\mathbf{y}}(n) \mathbf{f}^{H}(n) ; \\
& \mathbf{\Lambda}_{s}(n)=\operatorname{diag}\left(\mathbf{R}_{B}(n) \boldsymbol{\theta}_{A}(n) \boldsymbol{\theta}_{A}^{H}(n) \mathbf{R}_{B}^{H}(n)\right) .
\end{aligned}
$$

\section{NUMERICAL RESULTS}

Numerical simulations have been implemented to illustrate the performance of the group-blind and blind multiuser detector in MC-CDMA systems. We consider a synchronous MC-CDMA system in uplink channels with random spreading sequence. The input SNR is defined as the average transmitted bit energy to noise ratio $P_{k} / \sigma_{v}^{2}$. The multiple access interferers (MAI's) are 10-dB, i.e., $P_{k} / P_{0}=10$. The scalar ambiguity of the blind channel estimator is compensated with a complex constant, which makes the first element of the estimation $\hat{\mathbf{h}}_{0, p}$ and that of the true channel vector $\mathbf{h}_{0, p}$ identical.

First, the steady-state performance of the detectors is investigated. In the case of static channel, we set $M=32$ and $P=3$. There are $K=10$ users, of where $\hat{K}=7$ are known users. A multipath channel with six paths is considered. The channel coefficients are randomly generated according to a complex Gaussian distribution. The simulation results are averaged over 200 Monte-Carlo trials. Fig. 2(a) and (b) illustrate the steady-state signal-to-interference-plus ratio (SINR) of the subspace-based group-blind, blind MMSE receivers and the training MMSE detector versus data samples and the input SNR. Assume that the transmitting symbols among the users are zero mean and i.i.d. sequences. Consequently, we have

$$
\mathbf{R}_{\mathbf{y}}=P_{0} \mathbf{d}_{0} \mathbf{d}_{0}^{H}+\left(\sum_{k=1}^{K-1} P_{k} \mathbf{d}_{k} \mathbf{d}_{k}^{H}+\sigma_{n}^{2} \mathbf{I}\right)=\mathbf{R}_{s}+\mathbf{R}_{i n} .
$$



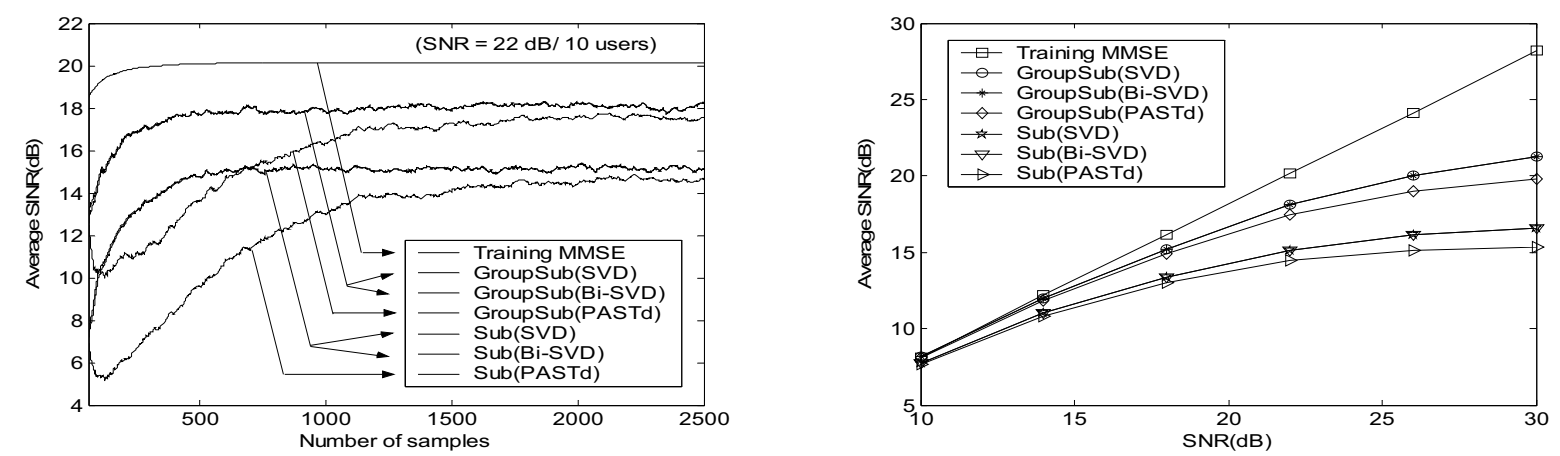

Fig. 2. Output SINR for the group-blind, blind detectors and training MMSE detector versus: (a) data samples. (b) input SNR. $M=32, P=3, K=10 / \hat{K}=7, \lambda=0.995$, and $\delta=0.1$.

The output SINR is defined as $\operatorname{SINR}=\left(\mathbf{w}^{H} \mathbf{R}_{s} \mathbf{w}\right) /\left(\mathbf{w}^{H} \mathbf{R}_{\text {in }} \mathbf{w}\right)$. It shows that the group-blind detection substantially outperforms the blind receiver in terms of output SINR. In the simulations, the initial estimation of the signal subspace required for the PASTd is obtained by applying an SVD to the first 50 data vectors. It can be seen that the convergence of the PASTd is rather slow even with this initialization. Whereas, the Bi-SVD presents much faster convergence, and its performance is very close to that of the detectors using an exact SVD.

Next, a slowly time-varying channel with $L=6$ Rayleigh fading paths in indoor environments is considered, where the multipath intensity profile decays exponentially. The maximum Doppler frequency $\left(f_{D}\right)$ is $52 \mathrm{~Hz}$ corresponding to a terminal speed $v=3 \mathrm{~m} / \mathrm{s}$ with a carrier frequency of $5.2 \mathrm{GHz}$. The subcarrier spacing ( $\Delta f$ ) is $312.5 \mathrm{KHz}$, and the time duration at the subcarrier is $T_{s}=3.2 \mu \mathrm{s}$. Hence, the normalized fading rate is $f_{D} T_{S}=1.664 \times 10^{-4}$. Due to the slow convergence of the PASTd, the Bi-SVD is used for signal subspace tracking. We average simulation results over 500 Monte-Carlo trials. Fig. 3 depicts the BER comparison for the subspace-based group-blind and blind receivers. To save simulation time, we set $M=16$ and $P=8$. There are $K=7$ users, of which $\hat{K}=5$ are known users Again, it is seen that the group-blind detector performs much better than the blind linear detector. Moreover, the performance of the blind multiuser detectors with signal and noise subspace tracking is very close to that of the receivers using an exact SVD.

\section{CONCLUSIONS}

In this paper, a group-blind detector for MC-CDMA systems is studied. By imposing linear vector constraints, the group-blind detector zero-forces the interferers within the cell, and suppresses the unknown interferes from other cells by MMSE criteria. Hence, it considerably outperforms the subspace-based blind detector. Signal and noise subspace tracking algorithms are investigated to recursively updating the coefficients of the group-blind and blind detectors. Numerical results show that the group-blind detector performs much better than the blind detector, and the performance of detectors using the Bi-SVD is very close to that of detectors using an exact SVD.

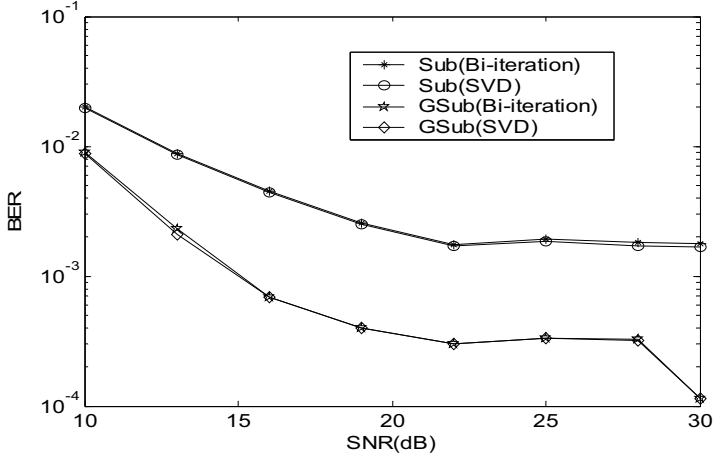

Fig. 3. BER comparison for the group-blind and blind detectors. $M=16, P=8, K=7 / \hat{K}=5, \lambda=0.96$, and $\delta=0.1$.

\section{REFERENCES}

[1] N. Yee, J. P. Linnartz, and G. Fettweis, "Multicarrier CDMA in indoor wireless radio networks", PIMRC, pp. 109-133, vol. 1, 1993.

[2] S. Hara and R. Prasad, "Overview of multicarrier CDMA", IEEE Commun. Magazine, vol. 35, pp. 126-133, Dec. 1997.

[3] X. D. Wang and H. Vincent, "Blind multiuser detection: A subspace approach", IEEE Trans. Inform. Theory, vol. 44, pp. 677690, March 1998.

[4] X. D. Wang and A. Host-Madsen, "Group-blind multiuser detection for uplink CDMA", IEEE J. Select. Areas Commun., vol. 17, pp. 1971-1984, Nov. 1999.

[5] J. Namgoong, T.F. Wong and J.S. Lehnert, "Subspace MMSE receiver for multicarrier CDMA", IEEE WCNC '99, pp. 90-94, New Orleans, LA, 1999.

[6] W. Sun and H. B. Li, "“Blind channel identification for multicarrier CDMA systems with transmit diversity", ICC'02, pp. 727731, NY, 2002."

[7] P. Comon and G. H. Golub, "Tracking a few extreme singular values and vectors in signal processing", Proc. IEEE, vol. 78, pp. 1327-1343, Aug. 1990.

[8] P. Strobach, "Bi-iteration SVD subspace tracking algorithms", IEEE Trans. Signal Processing, vol. 45, pp. 1222-1240, May. 1997.

[9] B. Yang, "Projection approximation subspace tracking", IEEE Trans. Signal Processing, vol. 44, pp. 95-107, Jan. 1995.

[10] P. Strobach, "Square-root QR inverse iteration for tracking the minor subsapce", IEEE Trans. Signal Processing, vol. 48, pp. 29942999, Nov. 2000 